Revista Verde de Agroecologia e Desenvolvimento Sustentável

http://www.gvaa.com.br/revista/index.php/RVADS

ARTIGO CIENTÍFICO

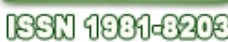

DOI: http://dx.doi.org/10.18378/rvads.v10i4.3396

\title{
Desenvolvimento e caracterização de salgadinho produzido a partir de gritz de milho nixtamalizado
}

\section{Development and characterization of snacks made from nixtamalized corn grits}

\author{
Laura Adriane de Moraes Pinto*, Fernanda de Oliveira Tavares, Mariana de Moraes Pinto, Alceu Kazuo Hirata, Gustavo \\ Affonso Pisano Mateus
}

\begin{abstract}
Resumo: Neste trabalho, buscou-se o enriquecimento nutricional de salgadinhos produzidos a partir da extrusão do gritz de milho, para tanto, duas diferentes formulações foram testadas. A primeira formulação consistiu em utilizar o gritz de milho aplicando-se o processo de nixtamalização; processo este conhecido pelo tratamento do gritz alcalinamente, utilizando o hidróxido de cálcio como agente de tratamento, enquanto que a segunda formulação, foi desenvolvida com gritz sem tratamento. Para a caracterização dos salgadinhos foram realizadas análises da composição centesimal e mineral, cor, textura e índice de expansão. Em relação à composição centesimal e concentração de cálcio, a amostra nixtamalizada apresentou diferença significativa para os parâmetros de cinzas, fibras e cálcio. Os valores obtidos para cinzas, foram de $0,44 \% \pm 0,02$ para os salgadinhos tratados e de $0,24 \% \pm 0,08$ para os não tratados. Para o teor de fibra os valores alcançados foram de $0,83 \% \pm 0,16$ e $0,40 \% \pm 0,03$, para os salgadinhos produzidos com tratamento e sem tratamento, respectivamente. Para o teor de cálcio, constatou-se um aumento superior a 6 vezes entre as formulações, em que $3,02 \pm 0,01\left(\mathrm{~g}_{\mathrm{Ca}} / \mathrm{Kg}_{\text {snack }}\right)$ foram obtidos para o salgadinho nixtamalizado e $0,49 \pm 0,003\left(\mathrm{~g}_{\mathrm{Ca}} / \mathrm{Kg}_{\text {snack }}\right)$ para os salgadinhos produzidos pelo gritz sem aplicação do tratamento. Os teores de umidade, proteínas e lipídeos, não apresentaram diferença significativa entre as formulações. A análise de cor mostrou que o salgadinho não sofreu influência do tratamento alcalino, porém a amostra tratada apresentou croma mais intensa. Já para a textura, a nixtamalização exerceu influência significativa para este parâmetro, em que os valores obtidos variaram de $1,95 \pm 0,49 \mathrm{~N}$ a $0,74 \pm 0,16 \mathrm{~N}$ para a amostra tratada e para a amostra sem tratamento, respectivamente. $\mathrm{O}$ índice de expansão (IE) está diretamente relacionado com a crocância dos salgadinhos, dessa maneira, o (IE) calculado neste trabalho, foi de 4,33 para os salgadinhos tratados e 4,43 para os não tratados alcalinamente, assim, a nixtamalização não interferiu significativamente na determinação deste parâmetro. Este estudo demonstrou que o tratamento alcalino do gritz de milho, seguido do processo de extrusão a alta temperatura, melhorou as características do salgadinho, uma vez que, houve um aumento do teor de cálcio e fibra presente na amostra avaliada.
\end{abstract}

Palavras-chaves: gritz, extrusão, hidróxido de cálcio.

Abstract: In this work, we sought to nutritional enrichment of chips produced from extruded corn grits, therefore, two different formulations were tested. The first formulation consisted of using corn grits applying the nixtamalization process; this known process by treating the alkali-grits using calcium hydroxide as the treatment agent, while the second formulation was developed with untreated grits. To characterize the snacks were performed analyzes of proximate and mineral composition, color, texture and expansion ratio. Regarding the chemical composition and concentration of calcium, nixtamalizada sample showed significant differences for the parameters of ash, fiber and calcium. The values obtained for ashes were $0.44 \pm 0.02 \%$ for the treated chips and $0.24 \pm 0.08 \%$ for the untreated. For the fiber obtained values were $0.83 \pm 0.16 \%$ and $0.40 \pm 0.03 \%$ for the snack foods produced with treatment and without treatment, respectively. To the calcium content, there was an increase of 6 times in the formulations, wherein $3.02 \pm 0.01\left(\mathrm{~g}_{\mathrm{C}} / \mathrm{Kg}_{\text {snack }}\right)$ were obtained for nixtamalizado snack and $0.49 \pm 0.003$ $\left(\mathrm{g}_{\mathrm{Ca}} / \mathrm{Kg}_{\text {snack }}\right)$ to the chips produced by the grits without the application of treatment. The moisture, protein and lipids, no significant differences between formulations. The color analysis showed that the snack not influenced by alkali treatment, but the treated sample had stronger chroma. As for the texture, nixtamalization in significant difference for this parameter, wherein the values obtained ranged from $1.95 \pm 0.49 \mathrm{~N} 0.74 \pm 0.16 \mathrm{~N}$ for the treated sample and the untreated sample, respectively. The expansion index (EI) is directly related to the crispness of snacks, this way, the (IE) calculated in this study, was 4.33 to 4.43 for snacks treated and untreated alkali-thus the nixtamalization did not interfere significantly in the determination of this parameter. This study demonstrated that the alkaline treatment of corn grits, followed by the process of high temperature extrusion, snack improved characteristics, since there was a increase in the calcium content and fiber present in the sample evaluated.

Key words: grits, extrusion, calcium hydroxide.

\footnotetext{
*Autor para correspondência

Recebido para publicação em 04/05/2015; aprovado em 29/09/2015

${ }^{1}$ Mestranda em Ciência de Alimentos, Universidade Estadual de Maringá, Maringá-PR; (44)8822-0177, E-mail: lauraampinto@ gmail.com.

${ }^{2}$ Mestranda em Engenharia Química, Universidade Estadual de Maringá, E-mail: fernandaoliveiratavares@gmail.com

${ }^{3}$ Graduanda em Engenharia Ambiental, Universidade Federal do Triângulo Mineiro, E-mail: mari.pinto05@gmail.com

${ }^{4}$ Mestrando em Zooctenia, Universidade Estadual de Maringá, E-mail: kzuhirata@ gmail.com

${ }^{5}$ Mestrando em Biotecnologia Ambiental, Universidade Estadual de Maringá, E-mail: gustavoapisano@gmail.com
} 


\section{INTRODUÇÃO}

Nos últimos anos o mercado de salgadinhos vem ganhando destaque, segundo a Associação Nacional das Indústrias de Biscoitos (ANIB), o segmento de snacks vem crescendo ano a ano, nos últimos dois anos foram lançados 1.136 novos snacks, sendo os salgadinhos de milho/trigo os que apresentaram o maior número de lançamentos (ABIMAP,2015).

A versatilidade de utilização do milho possibilita elevado emprego e o torna uma das matérias-primas mais importantes para a produção de alimentos (GONÇALVES et al.,2003).

Cada vez mais os salgadinhos vêm conquistando novos consumidores (FERREIRA, 2006). Snacks extrusados estão disponíveis numa grande variedade de formas, texturas e sabores, atributos que contribuem para sua alta aceitabilidade entre os consumidores (LIMBERGER et al.; 2009).

A nova geração de salgadinhos extrusados tenta promover não só a praticidade desse tipo de alimento, mas também associar a eles uma imagem de saúde através de novos produtos com baixos teores de gordura e sódio e altos teores de fibras (SOARES JÚNIOR et al.,2011).

A extrusão de alimentos se destaca entre as mais eficientes técnicas de cozimento, além de proporcionar alta produtividade e grande diversidade de produtos (FERNANDES et al., 2010). O processo de extrusão leva a mudanças reológicas e texturais dos grânulos de amido e constituintes, além do aumento da digestibilidade e disponibilidade como uma fonte de energia (CHEFTEL,1986).

Nixtamalização é um processo originário do México, utilizado para produzir muitos alimentos tradicionais, incluindo o mais popular e mais consumido de todos eles, as tortillas (MONDRAGÓN et al., 2004). Este processo se caracteriza pelo tratamento do milho em meio alcalino (MARTÍNEZ et al., 2010).

O tratamento do gritz de milho com hidróxido de cálcio, seguido de extrusão a alta temperatura, tem por finalidade melhorar a qualidade da proteína e disponibilizar e aumentar os teores de cálcio (FIGUEROA-CÁRDENAS et al., 2001 ).

O objetivo deste trabalho foi produzir um snack de milho extrusado, tratado com hidróxido de cálcio, a fim de melhorar suas características nutricionais.

\section{MATERIAL E MÉTODOS}

\section{Produção dos salgadinhos}

Os snacks foram produzidos utilizando gritz de milho em substituição ao milho in natura, comumente empregado no processo de nixtamilização.

O gritz de milho tipo canjiquinha, marca Zaeli ${ }^{\circledR}$, foi nixtamalizado de acordo com a metodologia descrita por Mora-Rochin et al. (2010) com modificações.

Inicialmente o gritz de milho foi adicionado de hidróxido de cálcio $(2,1 \%)$ e $(2 \%)$ de água destilada, homogeneizado por 30 minutos a $25^{\circ} \mathrm{C}$, em seguida o gritz foi embalado em saco de polietileno e armazenado à $4^{\circ} \mathrm{C}$ por 12 horas. Passado o tempo de armazenamento a amostra foi homogeneizada durante 3 horas, a $25^{\circ} \mathrm{C}$ e posteriormente extrusada para obtenção dos snacks. Paralelamente, seguindo as mesmas condições de homogeneização, armazenamento e extrusão, a amostra sem adição de hidróxido de cálcio, foi preparada para comparação dos resultados.

Para a caracterização dos salgadinhos foram realizadas análises físico-químicas: de textura, cor e índice de expansão. Os salgadinhos foram produzidos em linha completa de extrusão IMBRA-RX Imbramaq ${ }^{\circledR}$, com velocidade variável e capacidade de produção de $45 \mathrm{~kg} / \mathrm{hora}$. As condições de processamento foram: abertura da matriz de $3 \mathrm{~mm}$ e aquecimento $\pm 120^{\circ} \mathrm{C}$.

Após a extrusão, foi realizada a secagem em estufa de convecção de ar forçada à temperatura de $60^{\circ} \mathrm{C}$, por $8 \mathrm{~h}$. As amostras foram embaladas em sacos de polietileno e armazenadas à temperatura ambiente.

\section{Análise da composição centesimal e de minerais}

Os salgadinhos foram caracterizados em relação à umidade, cinzas, lipídeos e proteínas. As análises foram feitas conforme metodologia descrita pelo Instituto Adolf Lutz (1985).

A análise de fibra bruta foi realizada submetendo as amostras à digestão ácida, com solução de ácido sulfúrico $1,25 \%$, seguida por digestão alcalina com hidróxido de sódio $1,25 \%$ conforme descrito por Brasil (2005).

A quantificação do mineral cálcio foi realizada, em conforme o método AOAC Internacional (2005). Todas as análises foram realizadas em duplicatas.

\section{Análise da cor}

A cor foi verificada através do uso do colorímetro Minolta CR-400 $\AA$, sendo os resultados expressos em valores $\mathrm{L}^{*}, \mathrm{a}^{*} \mathrm{e} \mathrm{b}^{*}$, onde os valores de $\mathrm{L}^{*}$ (luminosidade ou brilho) variam do preto (0) ao branco (100), os valores de $a^{*}$ variam do verde (-60) ao vermelho (+60) e os valores de $b^{*}$ variam do azul ao amarelo, ou seja, de $-60 \mathrm{a}+60$, respectivamente. A análise foi realizada em duplicata.

\section{Textura, fraturabilidade e dureza}

Para analise da textura utilizou-se texturômetro TA.TX2 B (Texture Techologies Corp., Scarsdale, NY) e para a análise dos dados o software XTRAD. Os parâmetros utilizados na avaliação das amostras foram: velocidade de pré-teste, $2 \mathrm{~mm} . \mathrm{s}^{-1}$; velocidade de teste, $2 \mathrm{~mm} . \mathrm{s}^{-1}$; velocidade pós-teste, $2 \mathrm{~mm} . \mathrm{s}^{-1}$; distância percorrida pela probe, $25 \mathrm{~mm}$ e limiar de força foi de 20,4gf. Para cada Tratamento, realizaram-se dez repetições.

\section{Índice de expansão}

A determinação do índice de expansão consiste na razão entre o diâmetro da amostra extrusada $(\mathrm{mm})$ e o diâmetro da matriz $(\mathrm{mm})$. Foram 10 determinações para cada uma das amostras, considerado o valor do diâmetro o valor obtido pela média aritmética dos produtos expandidos dentro de cada tratamento, conforme a metodologia proposta por Gujska \& Khan (1991).

$$
\mathrm{IE}=\frac{\text { diâmetro da amostra extrusada }(\mathrm{mm})}{\text { Diâmetro da matriz }(\mathrm{mm})}
$$

\section{Análise Estatística}

Os resultados das características avaliadas foram submetidos à análise de variância e as médias, quando 
significativas, comparadas pelo teste de Tukey ao nível de significância de 5\%.

\section{RESULTADOS E DISCUSSÃO}

Os resultados obtidos na caracterização dos salgadinhos estão apresentados na Tabela 1.

Para o teor de cinzas, a amostra tratada com cálcio apresentou $0,44 \%$, enquanto que a sem tratamento, $0,24 \%$. Os valores encontrados apresentaram diferença significativa $(\mathrm{p}<0,05)$ entre amostras. Conforme os resultados obtidos, o tratamento alcalino promoveu um aumento no teor de cinzas, uma vez que este parâmetro está diretamente relacionado com a composição de minerais. Segundo Castro et al. (2009), o teor de cinzas no gritz de milho varia de $0,24 \%$ a $0,27 \%$, o que confirma os resultados encontrados neste trabalho.

O aumento nos teores de fibra é um resultado desejável na extrusão, devido ao aumento do teor de amido resistente (SOUZA et al., 2007). No presente trabalho, o processo de nixtamalização promoveu o aumento nas fibras dos snacks, o que pode ser confirmado através dos resultados encontrados, onde os teores de fibras dos salgadinhos tratados com hidróxido de cálcio apresentaram $0,83 \%$ enquanto que os sem tratamento apresentaram $0,40 \%$, apresentando diferença significativa de 5\%. Limberger et al (2009) em seu trabalho com salgadinhos extrusados de quirera de arroz, obteve valor superior, sendo este $4,18 \%$.

A composição mineral de cálcio foi de $3,02\left(\mathrm{~g}_{\mathrm{Ca}} / \mathrm{kg}_{\text {snack }}\right)$ para a amostra tratada alcalinamente e de $0,49\left(\mathrm{~g}_{\mathrm{Ca}} / \mathrm{kg}_{\text {snack }}\right)$ para a amostra sem tratamento, mostrando um aumento de cálcio 6 vezes superior em relação a sem tratamento, confirmando a diferença significativa $(\mathrm{p}<0,05)$ presente entre as formulações. $\mathrm{O}$ resultado obtido, evidencia $\mathrm{o}$ enriquecimento nutricional promovido pelo processo de nixtamalização. Castillo et al (2009) encontraram valores semelhantes a este estudo. Em sua pesquisa ao utilizar $1 \%$ de adição de cálcio obtiveram $1,12\left(\mathrm{~g}_{\mathrm{Ca}} / \mathrm{kg}_{\text {snack }}\right)$, valor este proporcional ao alcançado nesta pesquisa, ao utilizar $2 \%$ de adição de cálcio.

A umidade está diretamente relacionada com o índice de expansão, valores de umidade superiores a $10 \%$ podem interferir negativamente no processo de expansão dos snacks (MIKALOUSKI et al.,2013). Em relação a umidade, as amostras adicionadas de hidróxido de cálcio apresentaram $5,98 \%$ e as sem adição 5,88\%. Limberger et al. (2009) e Castillo et al (2009) encontraram valores superiores para seus extrusados, sendo estes de $9,4 \%$ e $7,23 \%$, respectivamente.

Para proteínas os valores obtidos foram de 7,20\% para os snacks nixtamalizados e de $7,12 \%$ para os snacks sem tratamento, não apresentando diferença significativa ao nível de $5 \%$ entre as formulações. Resultados obtidos por Bombo (2006) para seus snacks de milho, foram de 7,5\%, valores no mesmo intervalo de confiança dos encontrados neste trabalho.

Em relação aos lipídeos, os snacks tratados apresentaram $0,05 \%$ e os não tratados $0,04 \%$, não apresentando diferença significativa $(\mathrm{p}<0,05)$. Tanto os salgadinhos tratados com cálcio como os sem tratamento mostraram possuir um baixo teor de lipídeos. Soares Junior et al. (2011) relatou ter encontrado 3,2\% de lipídeos em seus salgadinhos enriquecidos com feijão e arroz.

O tratamento com hidróxido de cálcio afeta a cor, odor, sabor e textura dos produtos (MARTÍNEZ et al. 2010).

Os resultados obtidos para os parâmetros de cor das amostras estão apresentados na Tabela 2.

Para luminosidade $\left(\mathrm{L}^{*}\right)$, os valores encontrados foram de 74,77 para os snacks com adição de cálcio e de 76,53, para os snacks sem adição. Ambas amostras apresentaram elevada luminosidade $\left(\mathrm{L}^{*}\right)$, com a predominância na cor amarela $\left(\mathrm{b}^{*}\right)$ sobre a cor verde $\left(\mathrm{a}^{*}\right)$, mostrando assim, que não houve diferença significativa $(\mathrm{p}<0,05)$ deste parâmetro entre as amostras. Os valores encontrados foram superiores aos relatados por Madrigal-Sánchez et al. (2014), que obtiveram valores de luminosidade variando de 30,25 a 33,30, para suas tortilhas a base farinha de milho azul extrusado e nixtamalizado. Entretanto, mesmo não havendo diferença significativa entre as amostras para o atributo cor, a amostra tratada com cálcio apresentou valores de croma superiores em relação à amostra sem tratamento. Tal fato se associa as possíveis reações entre os pigmentos encontrados no grão de milho e o hidróxido de cálcio, que propiciam as reações de escurecimento, tais como: caramelização e reação de Maillard (GÓMEZ et al., 1987).

Segundo Capriles e Arêas (2012), um dos atributos mais importantes para a aceitação de snacks é a textura. Os parâmetros medidos na análise instrumental do perfil de textura indicaram uma força de compressão média de 1,95 N aplicada aos snacks tratados com hidróxido de cálcio, enquanto na amostra padrão este valor foi de $0,74 \mathrm{~N}$, com diferença significativa entre as amostras $(p<0,05)$. Este resultado indica que a presença de hidróxido de cálcio no produto aumentou a firmeza do mesmo. Singh et al. (2003) encontraram valores semelhantes em seu trabalho sobre as propriedades texturais de tortilhas, obtendo valores variando de 0,84-1,97 N, assim como Mikalosuki et al (2014) que relatou valores de 0,95 a $1,18 \mathrm{~N}$ para seus extrusados de milho.

A expansão de produtos extrusados depende do grau de gelatinização do amido e da taxa de evaporação da água no momento da saída do produto da extrusora. Geralmente, a expansão máxima é desejada para snacks extrusados, uma vez que produtos com grande expansão são mais crocantes (SILVA, 2010). A expansão dos snacks tratados com hidróxido de cálcio foi de 4,33 (mm), enquanto que para os salgadinhos sem adição de cálcio o valor encontrado foi de 4,43 (mm). Ao nível de significância de 5\%, não houve diferença significativa entre as amostras, indicando que o tratamento alcalino não afetou esse parâmetro. Os resultados obtidos neste trabalho se assemelham aos resultados encontrados por Lustosa e colaboradores (2010) em seu trabalho com snacks de farinha de mandioca enriquecidos com caseína e com os de Camargo et al. (2008) em seu estudo com biscoitos de polvilho azedo extrusados com fibras, onde os índices de expansão variaram de 3,29 a 4,56 e 3,5 a 5,7, respectivamente. 
Tabela 1 - Composição centesimal dos salgadinhos extrusados.

\begin{tabular}{ccccccc}
\hline & & \multicolumn{2}{c}{ COMPOSIÇÃO DOS SNACKS EXTRUSADOS } & & \multicolumn{2}{c}{ MINERAIS } \\
\hline AMOSTRA & Umidade (\%) & Cinzas (\%) & Proteínas (\%) & Lipídeos (\%) & Fibras $(\%)$ & ${\text { Cálcio }\left(\mathrm{g} . \mathrm{Kg}^{-1}\right)}$ \\
\hline $\mathrm{T}_{\mathrm{C}}$ & $5,98^{\mathrm{a}} \pm 0,01$ & $0,44^{\mathrm{a}} \pm 0,02$ & $7,20^{\mathrm{a}} \pm 0,22$ & $0,05^{\mathrm{a}} \pm 0,02$ & $0,83^{\mathrm{a}} \pm 0,16$ & $3,02^{\mathrm{a}} \pm 0,010$ \\
$\mathrm{~T}_{\mathrm{A}}$ & $5,88 \mathrm{a} \pm 0,36$ & $0,24^{\mathrm{b}} \pm 0,08$ & $7,12^{\mathrm{a}} \pm 0,01$ & $0,04^{\mathrm{a}} \pm 0,01$ & $0,40^{\mathrm{b}} \pm 0,03$ & $0,49^{\mathrm{b}} \pm 0,003$ \\
\hline
\end{tabular}

$\mathrm{T}_{\mathrm{C}^{-}}$amostra tradada com hidróxido de cálcio, $\mathrm{T}_{\mathrm{A}^{-}}$amostra sem adição de hidróxido de cálcio.

Médias seguidas da mesma letra, na coluna, não diferem entre si $(\mathrm{p}<0,05)$.

Tabela 2- Média dos parâmetros de cor das amostras de salgadinho.

\begin{tabular}{cccc}
\hline Amostras & $\mathrm{L}^{*}$ & $\mathrm{a}^{*}$ & $\mathrm{~b}^{*}$ \\
\hline $\mathrm{T}_{\mathrm{C}}$ & $74,77^{\mathrm{a}} \pm 1,94$ & $-3,18^{\mathrm{a}} \pm 0,66$ & $31,14^{\mathrm{a}} \pm 0,81$ \\
$\mathrm{~T}_{\mathrm{A}}$ & $76,53^{\mathrm{a}} \pm 1,42$ & $-4,10^{\mathrm{a}} \pm 0,38$ & $28,42^{\mathrm{a}} \pm 2,92$ \\
\hline
\end{tabular}

$\mathrm{T}_{\mathrm{C}^{-}}$amostra tradada com hidróxido de cálcio, $\mathrm{T}_{\mathrm{A}^{-}}$amostra sem adição de hidróxido de cálcio.

Médias seguidas da mesma letra, na coluna, não diferem entre si $(p<0,05)$.

\section{CONCLUSÕES}

A partir das amostras avaliadas, pôde-se concluir que tratamento alcalino do gritz de milho antes do processo de extrusão proporcionou um aumento significativo $(\mathrm{p}<0.05)$ nos teores de cinzas, fibras e cálcio, confirmando assim, que o processo de nixtamalização proporcionou uma melhora na qualidade nutricional dos salgadinhos. Os teores de umidade, proteína, lipídeos, não sofreram influência do tratamento, além de os parâmetros de cor e o índice de expansão não terem sido afetados.

\section{REFERÊNCIAS BIBLIOGRÁFICAS}

ABIMAPI, Associação Brasileira das Indústrias de Biscoito, Massas alimentícias, Pães e Bolos industrializados. Disponível em: <http:// http://abimapi.com.br/noticiasdetalhe.php?i=MTEyNw== $>$ Acesso em 29 jan. 2015.

AOAC - Association of Official Analytical Chemists. Official methods of analysis of AOAC International. 18 ed., Gaithersburg, Maryland: AOAC International, 2005. $1219 p$.

BRASIL. Ministério da Saúde. Agência Nacional de Vigilância Sanitária. Métodos Físico-Químicos para Análise de Alimentos. Diário Oficial da União Brasília: Ministério da Saúde, 2005. 1018p.

BOMBO, A. J. Obtenção e caracterização nutricional de snacks de milho (Zea mays L.) e linhaça (Linum usitatissimum L.). 2006. Dissertação (Mestrado em Saúde Pública) - Universidade de São Paulo, São Paulo, 2006.

CAMARGO, K. F.; LEONEL, M.; MISCHAN, M. M. Produção de biscoitos extrusados de polvilho azedo com fibras: efeito de parâmetros operacionais sobre as propriedades físicas. Ciênc. Tecnol. Aliment., v.28(3): p.586-591, 2008.

CAPRILES, V. D.; ARÊAS, J. A. G. Redução da razão comprimento/diâmetro da extrusora e aumento da aceitabilidade de snacks à base de amaranto. Brazilian Journal of Food Technology, v. 14, n. 1, p. 19-26, 2011.

CASTILlO, V. K. C.; OCHO, M. L. A.; FIGUEROA, C. J. D., DELGADO, L. E.; GALlEGOS, I. J. A.; MORALES, C. J. Efecto de la concentración de hidróxido de calcio y tiempo de cocción del grano de maíz (Zea mays L.) nixtamalizado, sobre las características fisicoquímicas y reológicas del nixtamal. ALAN, v.59, n.4, pp. 425-432, 2009.

CASTRO, M.V.L; NAVES, M. M. V.; OLIVEIRA, J. P.; FROES, L. O. Rendimento industrial e composição química de milho de alta qualidade protéica em relação a híbridos comerciais. Pesq. Agropec. Trop., v. 39, n. 3, p. 233-242, 2009.

CHEFTEL, J. C. Nutritional effects of extrusion cooking. Food Chemistry, v. 20,n. 4, p. 263-283, 1986.

FERNANDES, A. F. ; NASCIMENTO, T. A. ; PEREIRA, Joelma ; CARVALHO, C. W. P. ; LIMA, R. F. M. . Efeito da adição de concentrado proteico de soro de leite nas propriedades tecnológicas de extrudados expandidos (snacks) de milho. In: II Simpósio Internacional de Extrusão de Alimentos, 2010, Rio de Janeiro. Anais... Rio de Janeiro: Sociedade Brasileira de Frutas e Hortaliças, p. 1-8, 2010.

FERREIRA, R. E. Avaliação de parâmetros do processo de extrusão e do teor de farelo de trigo adicionado em características de "snacks" expandidos de milho. 2006. 162f. Tese (Doutorado em Tecnologia de Alimentos) - Faculdade de Engenharia de Alimentos, Universidade Estadual de Campinas, Campinas-SP, 2006.

FIGUEROA-CÁRDENAS J. D.; GODINEZ, M. G. A.; MÉNDEZ, N. L. V.; GUZMÁN, A. L.; ACOSTA, L. M. F.; HERNÁNDEZ, J. G. Fortification and evaluation of 
the nixtamal tortillas. Arch. Latinoam. Nutr. v.51, p.293-302. 2001.

GÓMEZ, M. H. ; ROONEY, L. W.; WANISKA, R. D.; PFLUG-FELDER, R. L. Dry maize masa flours for tortilla and snack production. Cereal. 1987.

GONCALVES, R.A; SANTOS, J. P.; TOMÉ, P. H. F.; PEREIRA, R.G.F.A; ASCHERI, J. L. R;ABREU,C. M. P. Rendimento e composição Química de cultivares de milho e moagem a seco e produção de grits. Ciênc.agrotec., Lavras. V.27, n.3, p.643-650, 2003.

GUJSKA, E.; KHAN, K. Functional properties of extrudates from high starch fractions of navy and pinto beans and corn meal blended with legume high protein fractions. Journal of Food Science, v. 56(178), n. 2, p. 431-435, 1991.

INSTITUTO ADOLFO LUTZ. Normas analíticas do Instituto Adolfo Lutz: Métodos químicos e físicos para análise de alimentos. 2. ed. São Paulo: IMESP, 1985. 533 p.

LIMBERGER, V. M.; COMARELA, C. G.; PATIAS, C. D.; BRUM, F. B.; EMANUELLI, T.; SILVA, L. P. Produção de salgadinho extrusado de quirera de arroz para uso na indústria de alimentos. Cienc. Rural, v. 39, 2009.

LUSTOSA, B. H. B.; LEONEL, M.; MISCHAN, M. M. Parâmetros de extrusão na produção de snacks de farinha de mandioca enriquecidos com caseína. Semina: Ciências Agrárias, v. 31, n. 1, p. 109-126, 2010.

MADRIGAL-SÁNCHEZ, M. A. ; RAMOS-QUINTIRO, A.; MARTÍNEZ-BUSTOS, F.; PIZARRO- MENDÉLEZ, C. O.; RUÍZ-GUTTIERREZ, M. G. Effect of different calcium sources on the antioxidant stability of tortilla chips from extruded and nixtamalized blue corn (Zea mays L.) flours. Food Sci. Technol, v.34(1), p.143-149, 2014.

MARTÍNEZ, D. C.; MORENO-RAMOS, C.; MARTÍNEZMANRIQUE, E.; MORENO-MARTÍNEZ, E.; MENDEZ-ALBORES, A. Evaluación nutricional y textural de tortillas de maíz-frijol blanco nixtamalizadas, Interciencia, v.35, p.828-832, 2010.

MIKALOUSKI, F. B. S.; MONTEIRO, A. G.; MARQUES, D. R.; MONTEIRO, C. C. F.; BENOSI, L. Influência da granulometria da matéria-prima na expansão de extrusados de milho. Braz. J. Food Technol. v. 17, n. 1, p. 28-32, 2014.

MORA-ROCHIN, S.; GUTIÉRREZ-URIBE, J. A.; SERNASALDÍVAR, S. O.; SÁNCHEZ-PEÑA, P.; REYESMORENO, C.; MILÁN-CARRILLO, J. Phenolic content and antioxidant activity of tortillas produced from pigmented maize processed by conventional nixtamalization or extrusion cooking. Journal of Cereal Science, v.52, p.502-508. 2010.

MONDRAGÓN, M.; BELLO-PERÉZ, L. A.; AGAMA, E.; MELO, A.; BETANCURANCONA, D.; PENÃ, J. L. Effect of nixtamalization on the modification of the crystalline structure of maize starch. Carbohydrate Polymers, v.55, p.411-418, 2004.

SILVA, E. M. M. Desenvolvimento e caracterização físicoquímica de extrudados de milho e feijão / Erika Madeira Moreira da Silva. 2010. 230 f.: Tese (Doutorado em Ciência e Tecnologia de Alimentos) Programa de Pós-Graduação em Ciência e Tecnologia de Alimentos, Universidade Federal Rural do Rio de Janeiro, Rio de Janeiro, 2010.

SOARES JUNIOR, M. S.; SANTOS, T. P. B.; PEREIRA, G. F.; MINAFRA, C. S.; CALIARI, M.; SILVA, F. A. Development of extruded snacks from rice and bean fragments, Semina: Ciências Agrárias, v. 32, n. 1, p. 191-200, 2011

SOUZA, L. B.; LEONEL, M.; MISCHAN, M. M. Efeito dos parâmetros de extrusão nas propriedades físicas de extrusados de misturas de fécula de mandioca e polpa cítrica. Alimentos e Nutrição, v.18 (1), p.83-91, 2007.

SINGH, R. K.; SINGH, G.; CHAUHAN, G. S. Textural properties of baked tortilla chips. LWT - Food Science and Technology, v.36, p. 463-466, 2003. 